\title{
AN ANALYSIS OF FREE UNDAMPED TRANSVERSAL VIBRATION OF A CANTILEVER BEAM WITH A C-SECTION VARYING ACROSS LENGTH
}

\author{
Gullapalli Naga Venkata Krishna Chaitanya ${ }^{1}$, Katkuri Vijay ${ }^{2}$ \\ ${ }^{I}$ Student, Space Engineering, Politecnico di Milano, Italy \\ ${ }^{2}$ Student, Space Engineering, Politecnico di Milano, Italy
}

\begin{abstract}
Vibration of a beam with a varying C-section across the length is investigated. Analytical solutions of undamped free vibration of the cantilever beam are obtained using MATLAB and validated using the commercial finite element software ANSYS. Weak form of the governing equation is used; considering the beam to be Euler-Bernoulli beam. A Ritz-Galerkin approximation is resorted to for the evaluation of resulting stiffness, mass matrix and transversal natural frequencies.
\end{abstract}

KeyWords: Cantilever beam, Ritz-Galerkin approximation, Varying C-section, Euler-Bernoulli beam, Transversal Vibration, MATLAB, ANSYS, Natural frequencies.

\section{INTRODUCTION}

Beams with uniformly varying cross sections are widely used in engineering applications to save weight and/or to satisfy the engineering requirements. A varying C-cross section across the length; not so widely discussed in the literature is preferred for analysis.

Galerkin finite element method used [1] for studying nonlinear vibrations of beams considered the transverse displacement term together with certain assumptions regarding the nature of the vibration.

An approximate solution presented in [2] used a Galerkin's procedure for a beam subjected to axial loads. Results provide a comparison of increasing rate of fundamental frequency with amplitude for beams with different end conditions.

Response of beams and plates undergoing large amplitude free oscillations wasinvestigated byreducing the governing partial differential equations and use of one term approximation and elliptic functions [4-5];while a RitzGalerkin procedure was employed to present equally good results [6].

A Euler -Bernoulli beam of varying C-section along the length is analyzed here using Ritz-Galerkin approximation to derive analytical solutions of undamped free transversal vibrations. The results are the validated with a finite element model using the commercial software ANSYS.

\section{ANALYTICAL MODEL}

The cantilever beam considered has

Length $=0.6 \mathrm{~m}$

Material Properties: Young's Modulus:210 GPa

Density : $7800 \mathrm{~kg} / \mathrm{m}^{3}$

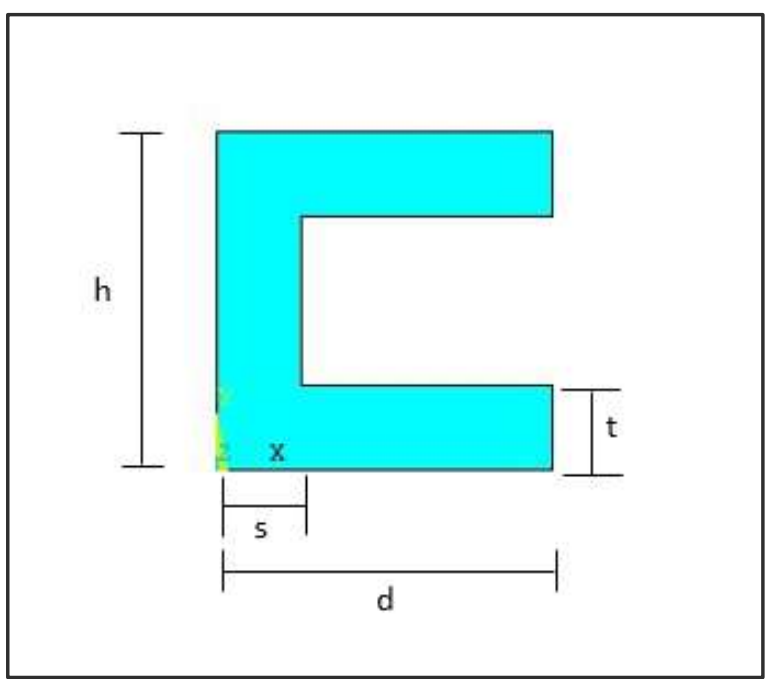

Fig -1: Dimensions of the C-section

Dimensions at Fixed end:

$$
\mathrm{h}=0.02 \mathrm{~m}, \mathrm{~s}=0.005 \mathrm{~m}, \mathrm{~d}=0.02 \mathrm{~m}, \mathrm{t}=0.005 \mathrm{~m}
$$

Dimensions at Free end

$$
\mathrm{h}=0.01 \mathrm{~m}, \mathrm{~s}=0.0025 \mathrm{~m}, \mathrm{~d}=0.01 \mathrm{~m}, \mathrm{t}=0.0025 \mathrm{~m}
$$


The principle of virtual work for the dynamic problem presented is

$$
\int_{0}^{l} \delta w_{/ x x}^{T} E J_{y}(x) w_{/ x x} d x=-\int_{0}^{l} \delta w^{T} m(x) \ddot{w} d x
$$

An approximate solution for the transverse displacement is

$$
w(x, t)=\sum_{i=1}^{N} N_{i}(x) u_{i}(t)
$$

where

$$
\begin{aligned}
N_{i}(x)=\frac{1}{i(i+1)(i+2)}\left(\frac{x}{l}\right)^{i+2} & \frac{1}{(i+1)(i+2)(i+3)}\left(\frac{x}{l}\right)^{i+3} \\
& +\frac{1}{2 i(i+1)}\left(\frac{x}{l}\right)^{i+2}
\end{aligned}
$$

is an approximate comparison function which satisfiesthe natural boundary conditions.

$$
\mathrm{w}(0)=0 ; \mathrm{w}_{/ \mathrm{x}}(0)=0 ; \mathrm{w}_{/ \mathrm{xx}}(\mathrm{l})=0 ; \mathrm{w}_{/ \mathrm{xxx}}(\mathrm{l})=0
$$

The element of the Mass Matrix is

$$
\begin{aligned}
& \frac{M(i, j)}{r h o * 10^{-4}} \\
& =2.5 l^{1}\left[\frac{1}{i j(i+1)(i+2)(j+1)(j+2)(i+j+5)}\right. \\
& -\frac{2 i j+3 i+3 j}{i j(i+1)(i+2)(i+3)(j+1)(j+2)(j+3)(i+j+6)} \\
& -\frac{1}{2 i j(i+1)(i+2)(j+1)(i+5)} \\
& +\frac{1}{(i+1)(i+2)(i+3)(j+1)(j+2)(j+3)(i+j+7)} \\
& +\frac{1}{2 j(i+1)(i+2)(i+3)(j+1)(i+6)} \\
& -\frac{1}{2 i j(i+1)(j+1)(j+2)(j+5)} \\
& +\frac{1}{2 i(i+1)(j+1)(j+2)(j+3)(j+6)} \\
& \left.+\frac{1}{20 i j(i+1)(j+1)}\right]
\end{aligned}
$$

$$
\begin{aligned}
& -3.3352 \mathrm{l}^{2}\left[\frac{1}{\mathrm{ij}(\mathrm{i}+1)(\mathrm{i}+2)(\mathrm{j}+1)(\mathrm{j}+2)(\mathrm{i}+\mathrm{j}+6)}\right. \\
& -\frac{2 \mathrm{j}+3 \mathrm{i}+3 \mathrm{j}}{\mathrm{ij}(\mathrm{i}+1)(\mathrm{i}+2)(\mathrm{i}+3)(\mathrm{j}+1)(\mathrm{j}+2)(\mathrm{j}+3)(\mathrm{i}+\mathrm{j}+7)} \\
& -\frac{1}{2 \mathrm{ij}(\mathrm{i}+1)(\mathrm{i}+2)(\mathrm{j}+1)(\mathrm{i}+6)} \\
& +\frac{1}{(\mathrm{i}+1)(\mathrm{i}+2)(\mathrm{i}+3)(\mathrm{j}+1)(\mathrm{j}+2)(\mathrm{j}+3)(\mathrm{i}+\mathrm{j}+8)} \\
& +\frac{1}{2 \mathrm{j}(\mathrm{i}+1)(\mathrm{i}+2)(\mathrm{i}+3)(\mathrm{j}+1)(\mathrm{i}+7)} \\
& -\frac{1}{2 \mathrm{ij}(\mathrm{i}+1)(\mathrm{j}+1)(\mathrm{j}+2)(\mathrm{j}+6)} \\
& +\frac{1}{2 \mathrm{i}(\mathrm{i}+1)(\mathrm{j}+1)(\mathrm{j}+2)(\mathrm{j}+3)(\mathrm{j}+7)} \\
& +\frac{1}{24 \mathrm{ij}(\mathrm{i}+1)(\mathrm{j}+1)]} \\
& +1.0430 \mathrm{l}^{3}\left[\frac{1}{\mathrm{ij}(\mathrm{i}+1)(\mathrm{i}+2)(\mathrm{j}+1)(\mathrm{j}+2)(\mathrm{i}+\mathrm{j}+7)}\right. \\
& +\frac{1}{2 \mathrm{ij}+3 \mathrm{i}+3 \mathrm{j}} \\
& +\frac{1 \mathrm{ij}(\mathrm{i}+1)(\mathrm{i}+2)(\mathrm{i}+3)(\mathrm{j}+1)(\mathrm{j}+2)(\mathrm{j}+3)(\mathrm{i}+\mathrm{j}+8)}{2 \mathrm{ij}(\mathrm{i}+1)(\mathrm{j}+1)(\mathrm{j}+2)(\mathrm{j}+7)} \\
& +\frac{1 \mathrm{i}+1)(\mathrm{j}+1)(\mathrm{j}+2)(\mathrm{j}+3)(\mathrm{j}+8)}{2 \mathrm{ij}(\mathrm{i}+1)(\mathrm{i}+2)(\mathrm{j}+1)(\mathrm{i}+7)} \\
& +\frac{1}{2 \mathrm{i}+1)(\mathrm{i}+2)(\mathrm{i}+3)(\mathrm{j}+1)(\mathrm{j}+2)(\mathrm{j}+3)(\mathrm{i}+\mathrm{j}+9)}
\end{aligned}
$$

The element of the stiffness Matrix is

$$
\begin{aligned}
\frac{K(i, j)}{E * 10^{-8}}=\frac{1.208}{l^{3}} & {\left[\frac{1}{\mathrm{ij}(\mathrm{i}+\mathrm{j}+1)}\right.} \\
& -\frac{\mathrm{i}+\mathrm{j}+2 \mathrm{ij}}{\mathrm{ij}(\mathrm{i}+1)(\mathrm{j}+1)(\mathrm{i}+\mathrm{j}+2)} \\
& -\frac{1}{\mathrm{ij}(\mathrm{j}+1)(\mathrm{i}+1)} \\
& +\frac{1}{(\mathrm{i}+1)(\mathrm{j}+1)(\mathrm{i}+\mathrm{j}+3)} \\
& +\frac{1}{\mathrm{j}(\mathrm{i}+1)(\mathrm{j}+1)(\mathrm{i}+2)} \\
& -\frac{1}{\mathrm{ij}(\mathrm{i}+1)(\mathrm{j}+1)} \\
& +\frac{1}{\mathrm{i}(\mathrm{i}+1)(\mathrm{j}+1)(\mathrm{j}+2)} \\
& \left.+\frac{1}{\mathrm{ij}(\mathrm{i}+1)(\mathrm{j}+1)}\right]
\end{aligned}
$$




$$
\begin{aligned}
& -\frac{3.5428}{l^{2}}\left[\frac{1}{i j(i+j+2)}-\frac{i+j+2 i j}{i j(i+1)(j+1)(i+j+3)}\right. \\
& -\overline{\mathrm{ij}(\mathrm{j}+1)(\mathrm{i}+2)} \\
& +\frac{1}{(i+1)(j+1)(i+j+4)} \\
& +\frac{1}{\mathrm{j}(\mathrm{i}+1)(\mathrm{j}+1)(\mathrm{i}+3)} \\
& -\frac{1}{\mathrm{ij}(\mathrm{i}+1)(\mathrm{j}+2)} \\
& +\frac{1}{i(i+1)(j+1)(j+3)} \\
& \left.+\frac{1}{2 \mathrm{ij}(\mathrm{i}+1)(\mathrm{j}+1)}\right] \\
& +\frac{3.8219}{l^{1}}\left[\frac{1}{i j(i+j+3)}-\frac{i+j+2 i j}{i j(i+1)(j+1)(i+j+4)}\right. \\
& -\overline{\mathrm{ij}(\mathrm{j}+1)(\mathrm{i}+3)} \\
& +\frac{1}{(i+1)(j+1)(i+j+5)} \\
& +\frac{1}{\mathrm{j}(\mathrm{i}+1)(\mathrm{j}+1)(\mathrm{i}+4)} \\
& -\frac{1}{\mathrm{ij}(\mathrm{i}+1)(\mathrm{j}+3)} \\
& +\frac{1}{\mathrm{i}(\mathrm{i}+1)(\mathrm{j}+1)(\mathrm{j}+4)} \\
& \left.+\frac{1}{3 \mathrm{ij}(\mathrm{i}+1)(\mathrm{j}+1)}\right] \\
& -\frac{1.7862}{1}\left[\frac{1}{i j(i+j+4)}-\frac{i+j+2 i j}{i j(i+1)(j+1)(i+j+5)}\right. \\
& -\frac{1}{\mathrm{ij}(\mathrm{j}+1)(\mathrm{i}+4)} \\
& +\frac{1}{(i+1)(j+1)(i+j+6)} \\
& +\frac{1}{j(i+1)(j+1)(i+5)} \\
& -\frac{1}{\mathrm{ij}(\mathrm{i}+1)(\mathrm{j}+4)} \\
& +\frac{1}{i(i+1)(j+1)(j+5)} \\
& \left.+\frac{1}{4 \mathrm{ij}(\mathrm{i}+1)(\mathrm{j}+1)}\right]
\end{aligned}
$$

$$
\begin{aligned}
& +\frac{0.30185}{l^{-1}}\left[\frac{1}{i j(i+j+5)}-\frac{i+j+2 i j}{i j(i+1)(j+1)(i+j+6)}\right. \\
& -\frac{1}{i j(j+1)(i+5)} \\
& +\frac{1}{(i+1)(j+1)(i+j+7)} \\
& +\frac{1}{j(i+1)(j+1)(i+6)} \\
& -\frac{1}{\mathrm{ij}(\mathrm{i}+1)(\mathrm{j}+5)} \\
& +\frac{1}{\mathrm{i}(\mathrm{i}+1)(\mathrm{j}+1)(\mathrm{j}+6)} \\
& \left.+\frac{1}{5 i j(i+1)(j+1)}\right]
\end{aligned}
$$

The convergence of the solution and the natural frequencies for the first 3 transversal modes are:

Table -1: Transversal Natural frequencies and convergence using MATLAB

\begin{tabular}{|l|l|l|l|}
\hline $\begin{array}{l}\text { Number } \\
\text { of terms }\end{array}$ & \multicolumn{1}{|c|}{ Mode 1 } & \multicolumn{1}{|c|}{ Mode 2 } & \multicolumn{1}{c|}{ Mode 3 } \\
\hline 1 & 72.3 & - & - \\
\hline 2 & 68.1 & 365.2 & - \\
\hline 3 & 67.8 & 310.8 & 1022.7 \\
\hline 4 & 67.8 & 305.6 & 797.5 \\
\hline 5 & 67.8 & 305.3 & 774.3 \\
\hline 6 & 67.8 & 305.3 & 773.8 \\
\hline 7 & 67.8 & 305.3 & 773.8 \\
\hline
\end{tabular}




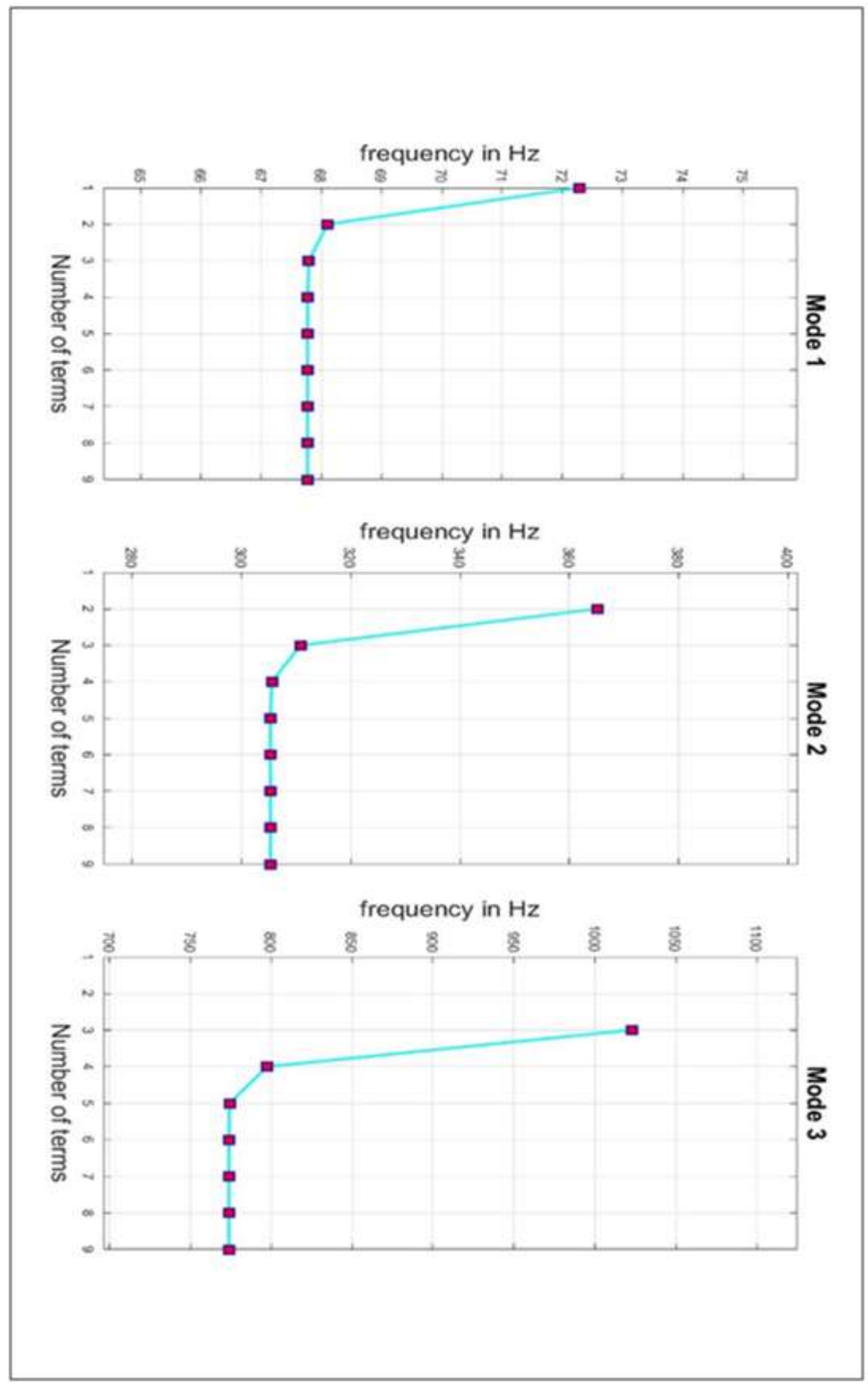

Fig -2: Convergence of solution 


\section{FINITE ELEMENT ANALYSIS}

The results of the finite element model built using solid45 elements are plotted below.

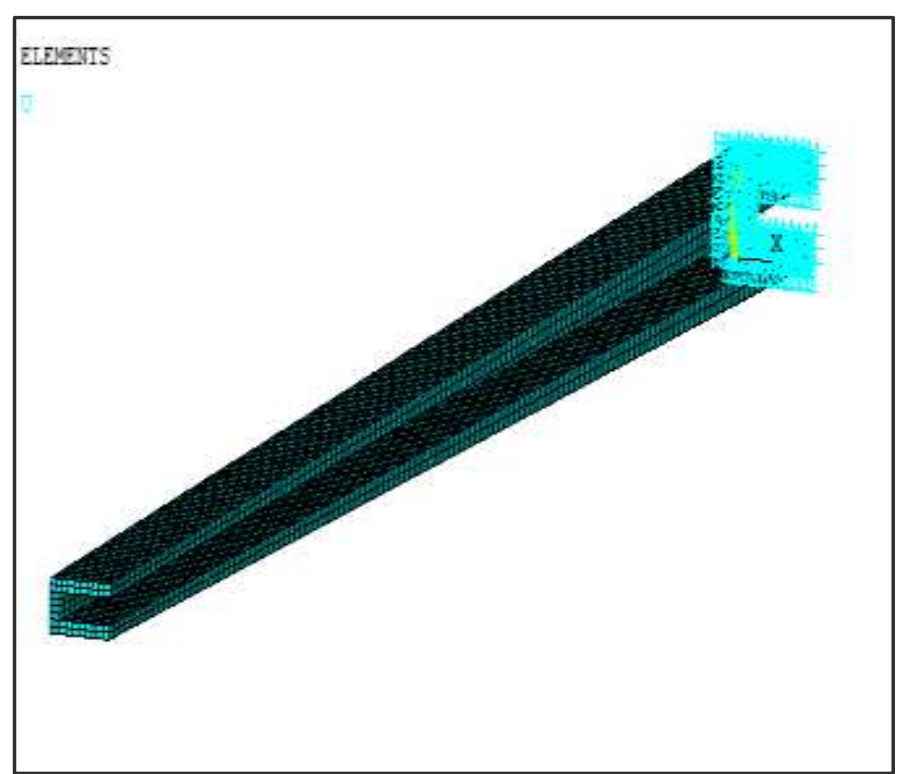

Fig -3: FEM of cantilever beam with varying C-section

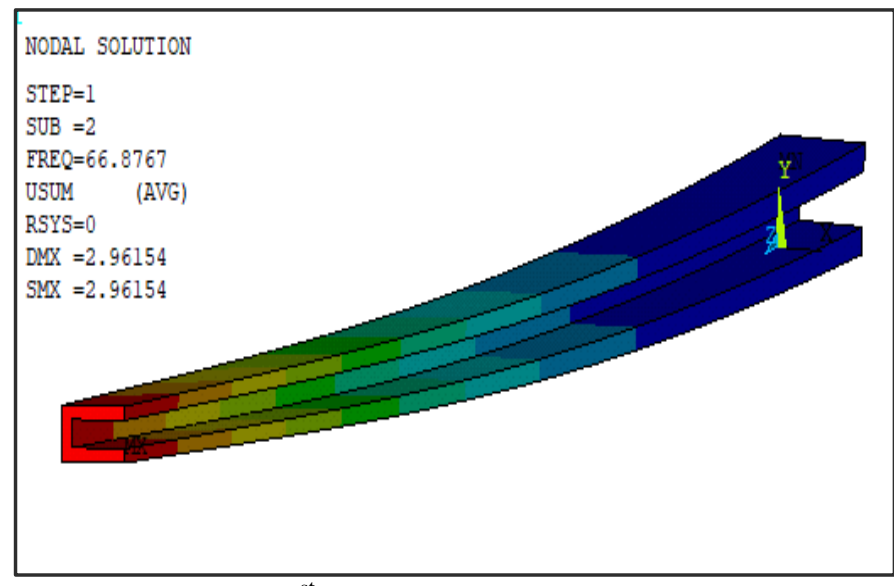

Fig $-4: 1^{\mathrm{st}}$ Transversal bending mode

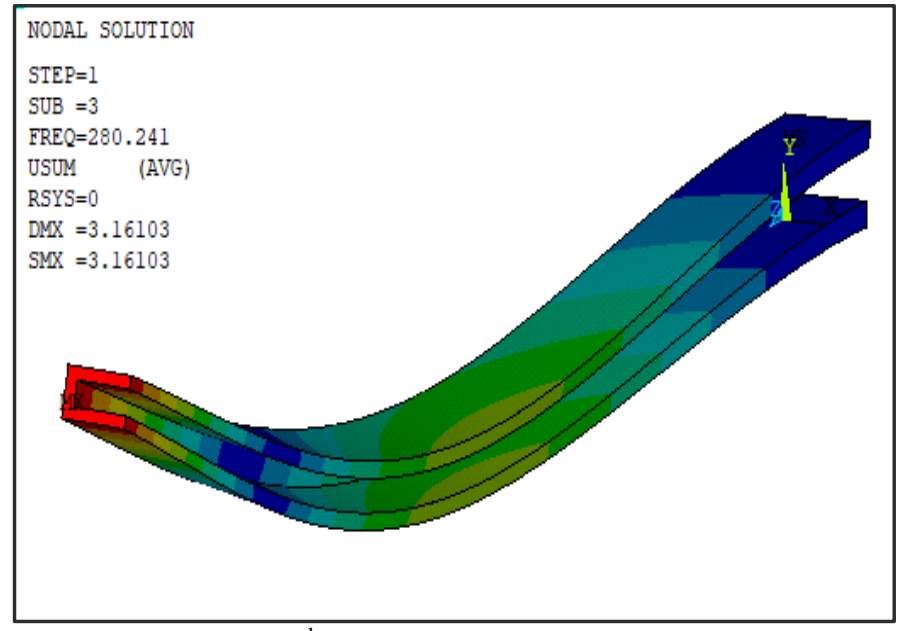

Fig -5: $2^{\text {nd }}$ Transversal bending mode

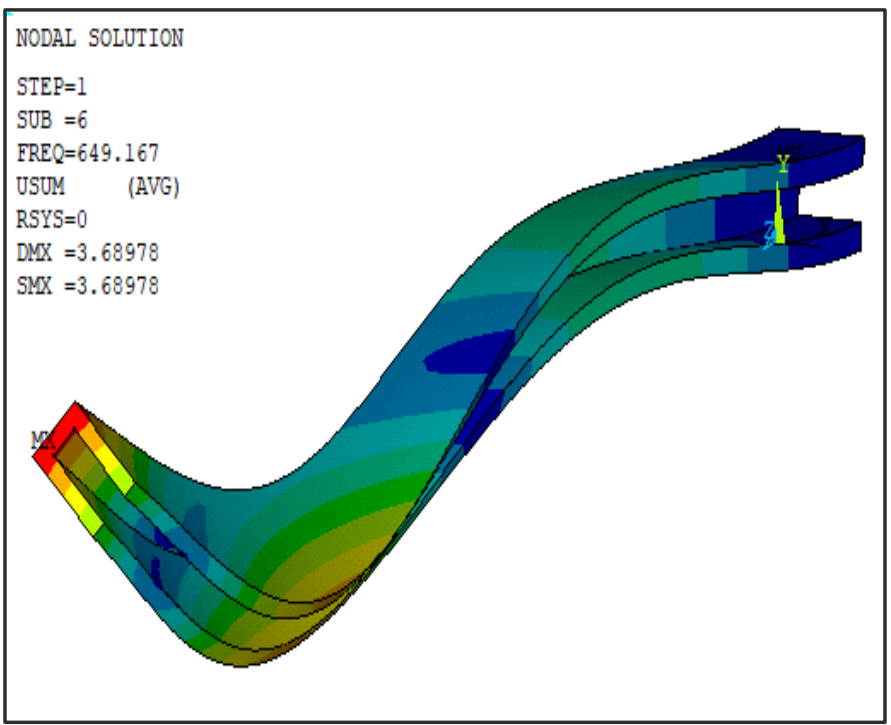

Fig -6: $3^{\text {rd }}$ Transversal bending mode

\section{CONCLUSION}

Table -2: Comparison of results

\begin{tabular}{|l|l|l|l|}
\hline & Mode 1 & Mode 2 & Mode 3 \\
\hline Ritz-Galerkin & 67.8 & 305.3 & 773.8 \\
\hline FEM & 66.8 & 280.2 & 649.1 \\
\hline Error \% & 1.47 & 8.22 & 16.11 \\
\hline
\end{tabular}

It can be concluded that the Ritz Galerkin approach using a Euler Bernoulli beam is accurate in predicting the first transversal bending mode while there is considerable error in the $2^{\text {nd }}$ and $3^{\text {rd }}$ mode as it neglects any complicated effects that arise from inhomogeneous mass distribution, variable cross section, non-uniform bending stiffness.

\section{ACKNOWLEDGEMENT}

We acknowledge our family, friends and professors for the unwavering love, support and encouragement.

\section{REFERENCES}

[1] Bhashyam, G. R.; Prathap, G.: Galerkin finite element method for non-linear beam vibrations. J. Sound Vib. 72 (1980) 191-203

[2] Sato, K.: Nonlinear free vibrations of beams with clamped ends and with one end clamped, other end simply supported. Bull. JapanSoe. Mech. Eng. 11 (1968) 1027-1036

[3] Nash, W. A. and Modeer, J. R., Certain approximate analysis of the nonlinear behavior of plates and shallow shells, Proceedings of the Symposium on the Theory of Thin Elastic Shells (North-Holland Publishing Co., Amsterdam, 1959)

[4] Wah, T., Vibration of circular plates at large amplitudes,J. Eng. Mech. Div., Am. Soc. Civil Engrs. 89 (October 1963), 1-15 
[5] Eisley, J. G., Nonlinear vibration of beams and rectangular platesZ. Angew. Math. Phys. 15 (March 1964), 167-168

[6] Srinivasan A.V, Large amplitude free oscillations of beams and plates. American Institute of Aeronautics and Astronautics Journal 3 (1965), 1951-1953.

[7] Rao S.S,vibration of continuous systems, 2007

[8] Leonard Meirovitch, Fundamentals of vibration,2010 